\title{
Unveiling the immunomodulatory properties of Haemonchus contortus adhesion regulating molecule 1 interacting with goat $T$ cells
}

Mingmin Lu', Xiaowei Tian ${ }^{1}$, Yang Zhang ${ }^{1}$, Kalibixiati Aimulajiang ${ }^{1}$, Wenjuan Wang ${ }^{1}$, Muhammad Ehsan ${ }^{1}$, Charles Li ${ }^{2}$, Ruofeng Yan' ${ }^{1}$, Lixin Xu' ${ }^{1}$, Xiaokai Song ${ }^{1}$ and Xiangrui Li ${ }^{1 *}$

\begin{abstract}
Background: Gastrointestinal nematodes could release excretory-secretory (ES) proteins into the host environment to ensure their survival. These ES proteins act as immunomodulators to suppress or subvert the host immune response via the impairment of immune cell functions, especially in chronic infections. In our preliminary study, Haemonchus contortus adhesion-regulating molecule 1 (HCADRM1) was identified from H. contortus ES proteins (HcESPs) that interacted with host T cells via liquid chromatography-tandem mass spectrometry analysis. However, little is known about HCADRM1 as an ES protein which may play a pivotal role at the parasite-host interface.
\end{abstract}

Methods: Based on bioinformatics approaches, multiple amino acid sequence alignment was conducted and the evolutionary relationship of HCADRM1 with ADRM1 orthologues was extrapolated. Employing RT-qPCR and immunohistochemistry assays, temporal transcriptional and spatial expression profiles of HCADRM1 were investigated. Using immunostaining approaches integrated with immunological bioassays, the immunomodulatory potentials of HCADRM1 on goat T cells were assessed.

Results: We hereby demonstrated that HCADRM1 with immunodiagnostic utility was a mammalian ADRM1 orthologue abundantly expressed at all developmental stages of $H$. contortus. Given the implications of ADRM1 proteins in cell growth, survival and development, we further investigated the immunomodulatory property of HcADRM1 as an individual ES protein acting at the parasite-host interface. The rHCADRM1 stimuli notably suppressed T cell viability, promoted intrinsic and extrinsic T cell apoptosis, inhibited T cell proliferation and induced cell cycle arrest at G1 phase. Simultaneously, rHCADRM1 stimuli exerted critical controls on T cell cytokine secretion profiles, predominantly by restraining the secretions of interleukin (IL)-4, IL-10 and interferon-gamma.

Conclusions: Importantly, HCADRM1 protein may have prophylactic potential for anti-H. contortus vaccine development. Together, these findings may contribute to the clarification of molecular and immunomodulatory traits of ES proteins, as well as improvement of our understanding of parasite immune evasion mechanism in H. contortus-host biology.

Keywords: H. contortus, Excretory-secretory protein, Adhesion-regulating molecule 1 (ADRM1), Immunomodulation, Immune evasion

\footnotetext{
*Correspondence: lixiangrui@njau.edu.cn

${ }^{1} \mathrm{MOE}$ Joint International Research Laboratory of Animal Health and Food

Safety, College of Veterinary Medicine, Nanjing Agricultural University,

Nanjing 210095, Jiangsu, People's Republic of China

Full list of author information is available at the end of the article
}

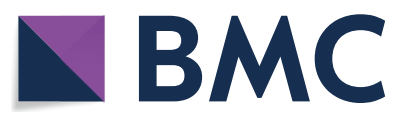

(c) The Author(s) 2020. This article is licensed under a Creative Commons Attribution 4.0 International License, which permits use, sharing, adaptation, distribution and reproduction in any medium or format, as long as you give appropriate credit to the original author(s) and the source, provide a link to the Creative Commons licence, and indicate if changes were made. The images or other third party material in this article are included in the article's Creative Commons licence, unless indicated otherwise in a credit line to the material. If material is not included in the article's Creative Commons licence and your intended use is not permitted by statutory regulation or exceeds the permitted use, you will need to obtain permission directly from the copyright holder. To view a copy of this licence, visit http://creativeco mmons.org/licenses/by/4.0/. The Creative Commons Public Domain Dedication waiver (http://creativecommons.org/publicdomain/ zero/1.0/) applies to the data made available in this article, unless otherwise stated in a credit line to the data. 


\section{Background}

The highly conserved and regulated ubiquitin (Ub) proteasome pathway is the primary mechanism for targeted elimination of most short-lived proteins including misfolded or damaged proteins in eukaryotic cells [1]. Ub can covalently attach to cellular proteins by Ub modification which is an ATP-dependent process mediated via different classes of Ub enzymes [2]. Alongside three families of shuttling factors $(\operatorname{Rad} 23$, Dsk2 and Ddi1), three proteasome subunits located in the sub-complex of $26 \mathrm{~S}$ proteasome, Rpn1, Rpn10 and Rpn13, are demonstrated to be Ub receptors as well. As the proteasome-associated polyubiquitin receptor, Rpn13, also termed as adhesion-regulating molecule 1 (ADRM1), is recruited by Rpn2 to be assembled into the $19 \mathrm{~S}$ regulatory particle and target protein substrates linked to the small protein Ub via its pleckstrin-like receptor [3, 4]. Simultaneously, the C-terminal adaptor domain of ADRM1 serves to bind and activate the deubiquitylase UCHL5/UCH37, and enhance its isopeptidase activity, revealing a mechanism to accelerate $\mathrm{Ub}$ chain disassembly [5-7].

With engagement in the Ub proteasome pathway that regulates a broad range of physiological functions, ADRM1 is implicated in multitudinous cellular processes such as cell growth, migration, survival and development, particularly in cancer cells [8]. Recent publications reveal that ADRM1 transcription is consistently elevated in ovarian, colorectal and gastric cancer tissues, and knockdown of ADRM1 expression in both human colon carcinoma and gastric cancer cell lines suppress cell migration and proliferation, and induces cell apoptosis [9-11]. Meanwhile, Fejzo et al. [12] demonstrated that overexpression of ADRM1 in ovarian cancer promoted cell growth and migration, whereas blocking its expression caused cell death. Given the association of amounting ADRM1 expression with the onset and progression of cancers, ADRM1 has been defined as a potential predictive and therapeutic target for clinical therapy [13]. Additionally, comparable expressions of ADRM1 have also been observed in several lymphocyte cell lines as well as endothelial cell lines, and similar physiological roles of ADRM1 are described through its excessive expression in skin endothelial cells that facilitates T lymphocyte adhesion [14].

In a previous study [15], we identified 114 Haemonchus contortus excretory-secretory (ES) proteins (HcESPs) that interacted with host $\mathrm{T}$ cells via liquid chromatography mass spectrometry (LC-MS/MS) analysis. Haemonchus contortus ADRM1 (HcADRM1) protein, a mammalian ADRM1 homologue, was ascertained among these interacting proteins [15]. Additionally, recombinant HcADRM1 (rHcADRM1) was recognized by serum samples obtained at Day $7,14,21,35,49,63$ and 85 post-infection, derived from experimentally $H$. contortus-infected goats. As a result of these observations, HcADRM1 with immunodiagnostic utility was fostered as a hallmark of $H$. contortus infection, and a serological diagnosis assay with high sensitivity and specificity was developed using HcADRM1 antigen [16]. Furthermore, our preliminary analysis showed that HcESPs stimuli notably induced intrinsic and extrinsic apoptosis, suppressed $\mathrm{T}$ cell proliferation, and caused cell cycle arrested. HcESPs consisted of multitudinous modulatory molecules such as kinases, phosphatases, hydrolases and proteases, where the pleiotropic effects were initiated by a cascade of individual ES components. Importantly, the exact molecules that modulated $\mathrm{T}$ cell immune response in the parasite-host interaction warrant further investigation. Given the functional diversity of ADRM1, and especially its engagement in cell proliferation and apoptosis, HcADRM1 might be one of these dominated proteins that exert critical controls on cellular survival and death of host key effector cells. Therefore, herein we aimed to further investigate the molecular traits of HcADRM1 and address its immunomodulatory roles at the parasite-host interface.

\section{Methods}

\section{Parasite, animals and cells}

The $H$. contortus strain was propagated via serial passages in nematode-free goats in the Animal Experimental Center, Faculty of Veterinary Medicine, Nanjing, China. The collection of eggs, L3, xL3, male and female adults was performed as previously described $[17,18]$. Sprague Dawley (SD) rats (SCXK 2008-0004) with a standard packing weight $(\sim 150 \mathrm{~g})$ were obtained from Jiangsu Experimental Animal Center (Nanjing, China). They were maintained in a microbe-free room with access to sterilized food and water ad libitum.

Local crossbred and healthy goats (5-6 months-old) were reared in individually ventilated cages to prevent accidental infection with nematodes. Alongside ad libitum access to water in pens, these goats were given hay and whole shelled corn daily. Peripheral venous blood samples were obtained by venipuncture as described elsewhere, as well as the isolation of goat peripheral blood mononuclear cells (PBMCs) [19]. Total $\mathrm{T}$ cells in goat PBMCs were sorted using a magnetic-activated cell sorting system (MACS; Miltenyi Biotech Inc, Auburn, CA, USA) as described elsewhere [20]. Briefly, every million PBMCs in $100 \mu$ l staining buffer were incubated with 10 $\mu \mathrm{l}$ mouse anti-bovine CD2 primary antibody (Bio-Rad, Kidlington, UK) which cross-react with goat CD2 T cells for $30 \mathrm{~min}$. After two washes in PBS, $1 \times 10^{7}$ of total PBMCs resuspended in $100 \mu \mathrm{l}$ staining buffer were labeled 
with $10 \mu \mathrm{l}$ anti-FITC MicroBeads (Miltenyi Biotech) at room temperature for $15 \mathrm{~min}$. Subsequently, PBMCs were loaded onto the MACS magnetic system (Miltenyi Biotech) for positive sorting based on the manufacturer's specifications. T cells were then adjusted to a density of $1 \times 10^{6}$ cells/ml in RPMI 1640 (Gibco, Grand Island, NY, USA) containing $10 \%$ heat-inactivated fetal calf serum (FCS; Gibco) and $100 \mathrm{U} / \mathrm{ml}$ penicillin, $100 \mathrm{mg} / \mathrm{ml}$ streptomycin (Gibco). The viability of $\mathrm{T}$ cells was $>95 \%$ as assessed by the trypan blue exclusion test. The $95 \%$ purity of separated $\mathrm{T}$ cells was validated via flow cytometric determination (Additional file 1: Figure S1). Three goats (biological replicates) were used in every experiment.

\section{Sequence alignment and phylogenetic analysis}

The cloning and amplification of the complete coding sequence of the HCADRM1 gene were performed as previously described [16]. The amplified HCADRM1 fragment was cloned into pET28a (+) vector (Invitrogen, Carlsbad, CA, USA) and validated by sequence analysis using BLAST. Multiple amino acid sequences of ADRM1 orthologs were aligned for comparison by the CLUSTAL OMEGA software [21]. The evolutionary analysis was conducted and extrapolated by MEGA X software using JTT matrix-based model and the Maximum Likelihood method [22]. With partial deletion option, positions with less than $80 \%$ site coverage were excluded prior to phylogenetic analysis. Based on the optimized evaluation of 1000 replicates for bootstrap support, the evolutionary tree of ADRM1 orthologues was generated with several designated and collapsed branches.

\section{Expression and purification of the rHCADRM1 protein}

The expression and purification of rHcADRM1 proteins were conducted as elsewhere described [23]. Briefly, Escherichia coli BL21 (DE3) cells containing the reconstructed pET28a-HcADRM1 plasmid were incubated with Luria-Bertini medium containing kanamycin $(100 \mu \mathrm{g} / \mathrm{ml}$; Sigma-Aldrich, St. Louis, MO, USA) and then stimulated by isopropyl- $\beta$-Dthiogalactopyranoside (Sigma-Aldrich) for the induction of rHcADRM1 expression. The rHcADRM1 protein fused with a histidine-tag was obtained from the supernatant of cell lysates via His-Trap HP purification columns (GE Healthcare, Piscataway, NJ, USA). rHcADRM1 proteins were resolved on $12 \%$ sodium dodecyl sulfate-polyacrylamide gel electrophoresis (SDS-PAGE) gels for size and purity validation, and the concentration was determined by a bicinchoninic acid (BCA) assay (Thermo Fisher Scientific, Rockford, IL, USA). Employing the DetoxiGel Affinity Pak prepacked columns (Thermo Fisher Scientific), rHcADRM1 proteins were exempt of lipopolysaccharide contamination. As rHcADRM1 protein was dissolved in PBS, PBS-treated $\mathrm{T}$ cells served as the control group $(0 \mu \mathrm{g} / \mathrm{ml})$ in functional assays. The purified $\mathrm{rHcADRM} 1$ was stored at $-80{ }^{\circ} \mathrm{C}$ until further analysis.

\section{Preparation of polyclonal antibody ( $\mathrm{pAb})$}

To obtain antigen-specific pAb, rHcADRM1 proteins $(300 \mu \mathrm{g})$ blended with Freund's complete adjuvant was administrated subcutaneously into SD rats. With a 2-week interval, booster immunizations with $300 \mu \mathrm{g}$ of rHcADRM1 proteins emulsified in Freund's incomplete adjuvant were administered four times. Seven days after the final boost, rat sera containing anti-rHcADRM1 pAb were harvested and kept at $-80{ }^{\circ} \mathrm{C}$ for further analysis. The sera harvested from $H$. contortus-infected goats (anti- $H$. contortus serum) were stored at the Veterinary Parasitology Teaching and Research Center of Nanjing Agricultural University, Nanjing, China.

\section{Immunoblot analysis}

rHcADRM1 and HcESPs were resolved on protein gels, respectively, and transferred onto nitrocellulose membranes. The blots were blocked using 4\% BSA in TRISbuffered saline- $0.1 \%$ Tween 20 (TBST) for $1 \mathrm{~h}$ at room temperature. The blots with the rHcADRM1 samples were probed with primary goat anti- $H$. contortus serum (1:500 in TBST) or normal goat serum (control) at $4{ }^{\circ} \mathrm{C}$ overnight, while the blots with the HcESPs samples were probed with primary rat anti-rHcADRM1 IgG (1:500 in TBST) or normal rat IgG (control). After five washes in TBST, the blots were incubated with horseradish peroxidase-coupled rabbit anti-goat or anti-rat IgG $(\mathrm{H}+\mathrm{L})$ secondary antibody (Sigma-Aldrich) in TBST (1:5000) for $1 \mathrm{~h}$ at $37^{\circ} \mathrm{C}$. The blots were then developed with 3,3'-diaminobenzidine (DAB; Sigma-Aldrich) for 3-5 min and visualized by using a ChemiDoc imaging system (Bio-Rad, Hercules, CA, USA).

\section{HCADRM1 transcription in $\mathrm{H}$. contortus life-cycle stages}

To detect mRNA expression of HcADRM1 in H. contortus life-cycle stages, total RNA of eggs, L3, xL3, female and male adults were extracted using Trizol (Invitrogen), and the resulting cDNAs were synthesized in accordance with the manufacturer's specifications. Employing specific primers for the $\beta$-tubulin gene (endogenous reference) [24] and target gene HcADRM1 (Additional file 2: Table S1), transcriptional analysis of the HcADRM1 gene was conducted by real-time PCR using the QuantStudio 3 System (Applied Biosystems, Carlsbad, CA, USA) with a standard protocol. The specificity of the primers was 
validated to ensure product purity via generation of a melt curve and the absence of primer dimers. The amplification efficiencies and correlation coefficients were verified to be stable and similar. Based on the $2^{-\Delta \Delta C q}$ method, the relative transcription levels of HcADRM1 were normalized on $\beta$-tubulin transcription. Each experiment was run in triplicate.

\section{Immunohistochemistry assays}

Freshly collected female and male adults were washed, dehydrated, fixed, embedded and cut into cryostat sections as previous described [25]. To minimize nonspecific binding, cryosections were treated with $10 \%$ normal goat serum in PBS containing 0.1\% Tween 20 (PBST) for $1 \mathrm{~h}$. Subsequently, cryosections were served with primary anti-rHcADRM1 IgG (1:200) or sham control IgG overnight at $4{ }^{\circ} \mathrm{C}$. Prior to DNA staining with 2-(4-Amidinophenyl)-6-indolecarbamidine dihydrochloride (DAPI; Sigma-Aldrich), cryosections were then incubated with Cy3-labeled goat anti-rat IgG (1:500; Beyotime Biotechnology, Shanghai, China) at $37{ }^{\circ} \mathrm{C}$ for $1 \mathrm{~h}$. Subsequently, the samples were immersed in antifade medium (Sigma-Aldrich) to prevent fluorescence fading for microscopic examination. Finally, the sections were imaged at $60 \times$ magnification using a LSM710 fluorescence microscope (Zeiss, Jena, Germany), and ZEN 2012 software (Zeiss) was used for the analysis of digital images.

\section{The interaction of HcADRM1 protein with T cells in vitro}

The interaction of HcADRM1 with goat $\mathrm{T}$ cells was investigated as previously described [26]. In brief, freshly sorted T cells were cultured with or without $5 \mu \mathrm{g} / \mathrm{ml}$ rHcADRM1 proteins for $2 \mathrm{~h}$ at $37^{\circ} \mathrm{C}$. After three washes, $4 \%$ paraformaldehyde-fixed $\mathrm{T}$ cells were permeabilized by $0.5 \%$ Triton X-100 in PBST and blocked with 4\% BSA in PBST for $30 \mathrm{~min}$. Subsequently, prior to the staining with the Cy3-coupled secondary antibody (1:500), T cells were treated by primary anti-HcADRM1 pAb (1:100) or normal rat IgG (control) in a humidified chamber at $37^{\circ} \mathrm{C}$ for $1 \mathrm{~h}$. Followed by five PBST washes, T cells were subjected to Gold Anti-fade mounting solution containing DAPI (Life Technologies, Eugene, OR, USA) for nuclear staining. Immunofluorescence-labeled cells were visualized at $100 \times$ magnification using a LSM780 confocal microscope (Zeiss, Jena, Germany); Zen 2012 software (Zeiss) was employed for the interpretation of digital images.

\section{Cell viability}

The modulatory effects of rHcADRM1 on goat $\mathrm{T}$ cell viability were determined using the cell counting kit-8 assay (CCK-8; Dojindo, Kumamoto, Japan) as previously described [27]. Fresh sorted goat $\mathrm{T}$ cells activated with concanavalin A (ConA, $5 \mu \mathrm{g} / \mathrm{ml})$ were incubated in the presence of various doses of rHcADRM1 proteins (0, 5, 10, 20 and $40 \mu \mathrm{g} / \mathrm{ml}$ ) at $37^{\circ} \mathrm{C}$. Following 24 h-stimulation, cell culture medium was incorporated with $10 \mu \mathrm{l}$ CCK-8 solution and incubated at $37{ }^{\circ} \mathrm{C}$ in the dark for $4 \mathrm{~h}$. Following incubation, optical density was measured at $450 \mathrm{~nm}$ (OD450) using a microplate reader (Bio-Rad, Hercules, California, USA). Three independent tests, each in triplicate, were performed.

\section{Cell apoptosis assay}

Flow cytometry assays were performed for $\mathrm{T}$ cell apoptosis determination using the Annexin V-PE kit (BD Biosciences, San Jose, CA, USA) as previously described [28]. In brief, freshly sorted $T$ cells were cultured in the presence of tested doses of rHcADRM1 proteins $(0,5,10$, 20 and $40 \mu \mathrm{g} / \mathrm{ml}$ ) followed by Annexin V and 7-aminoactinomycin D (7-AAD) staining based on the kit's specification. The PBS-stimulated $\mathrm{T}$ cells served as negative controls. Three individual tests, each in triplicate, were conducted.

\section{Cell proliferation assay}

Cell proliferation analysis was determined using the Alexa Fluor 647 Click-iT plus EdU flow cytometry kit (Thermo Fisher Scientific) via the measurement of DNA synthesis directly based on the manufacturer's instructions. After $12 \mathrm{~h}$ co-incubation, the cell culture was incorporated with 5-ethynyl-2'-deoxyuridine (EdU, $10 \mu \mathrm{M})$ for another $12 \mathrm{~h}$ incubation. Subsequently, T cells were harvested, fixed with $4 \%$ paraformaldehyde in PBS and permeabilized using the Click-iT saponin-based reagent, followed by Click-iT reaction to coupled EdU with Alexa Fluor 647 dye. After three washes with $3 \mathrm{ml}$ of 1\% BSA in PBS, T cells were treated with 7-AAD staining solution (BD Biosciences). Flow cytometry was used for the determination of EdU ${ }^{+}$cells in the population. Each experiment consisting of three replicates was run in triplicate.

\section{Cell cycle assay}

Flow cytometry assays were conducted for cell cycle determination using PI/RNase staining buffer (BD Biosciences) according to the manufacturer's DNA staining protocol. Following co-incubation with rHcADRM1 stimuli $(20 \mu \mathrm{g} / \mathrm{ml})$ for $24 \mathrm{~h}, \mathrm{~T}$ cells were harvested, washed and fixed with ice-cold $75 \%$ ethanol every $6 \mathrm{~h}$. After being frozen at $-20{ }^{\circ} \mathrm{C}$ for more than $2 \mathrm{~h}$, treated-T cells were washed twice with PBS to remove remaining ethanol and resuspended in PI/RNase staining buffer for flow cytometry analysis. Each experiment consisting of three replicates was run in triplicate. 


\section{Transcription analysis}

$\mathrm{T}$ cells treated with different concentrations of rHcADRM1 $(0,5,10,20$ and $40 \mu \mathrm{g} / \mathrm{ml})$ for $12 \mathrm{~h}$ were harvested for the transcription analysis of the cell apoptosis pathway, and T cells treated with $20 \mu \mathrm{g} / \mathrm{ml}$ of rHcADRM1 for $24 \mathrm{~h}$ were collected for transcription analysis of the cell cycle pathway. Cells were harvested for total RNA extraction and cDNA obtained by reverse-transcription PCR. Relative quantification of candidate gene expression was conducted using previously published primers [29-33] of endogenous reference and candidate genes (Additional file 2: Table S2). Based on the $2^{-\Delta \Delta C q}$ method, the relative levels of target gene transcription were normalized to reference gene expression. Each experiment consisting of three replicates was run in triplicate.

\section{Detection of cytokine secretions}

For the determination of cytokine secretion levels, freshly isolated $\mathrm{T}$ cells activated by ConA $(5 \mu \mathrm{g} / \mathrm{ml})$ were treated with or without rHcADRM1 $(0,5,10,20$ and $40 \mu \mathrm{g} / \mathrm{ml}$ ) for $24 \mathrm{~h}$. Cell culture medium was harvested and determined for cytokine secretion detection using goat enzyme-linked immunosorbent assay (ELISA) kits (Mlbio, Shanghai, China) according to the manufacturer's specifications. The limit of quantification dependent upon each analytic kit ranged from between 2 and $800 \mathrm{pg} / \mathrm{ml}$. Each experiment was run in triplicate.

\section{Statistical analysis}

One-way and two-way analysis of variance (ANOVA) with Dunnett's multiple comparison test, alongside the Student's t-test, were performed for statistical analysis using GraphPad Premier 8.0 software (GraphPad Prism, San Diego, CA, USA). Differences were regarded as statistically significant when $P$-values were $<0.05$. Data were denoted as minimum to maximum (all points) or mean \pm standard deviation (SD).

\section{Results}

\section{Sequence alignment and phylogenetic analysis}

The entire coding region of the HCADRM1 gene (1083 bp) was amplified from the cDNA of adult worms, encoding a 361-amino acid protein with an estimated molecular mass around $42 \mathrm{kDa}$. We then performed a sequence alignment of ADRM1 orthologues derived from GenBank on zebrafish, human, mouse, Caenorhabditis elegans and $H$. contortus using CLUSTAL OMEGA software. HcADRM1 protein showed a moderate degree of identity to zebrafish (44.79\%), human (44.69\%), mouse (46.15\%) and C. elegans (47.14\%) orthologs (Fig. 1a). In addition, we conducted an evolutionary analysis of
HcADRM1 using the Maximum Likelihood method involving 10 amino acid sequences. Phylogenetic analysis clearly showed an evolutionary relationship of HcADRM1 with other ADRM1 orthologues, revealing that HcADRM1 was closely related to the Teladorsagia circumcincta homologue but divergent from vertebrate sequences (Fig. 1b).

\section{Protein expression and immuno-blot analysis}

The rHcADRM1 protein fused with the histidine-tag was successfully obtained in the supernatant of cell lysates. After purification, rHcADRM1 was visualized by Coomassie Blue staining as a single band with a molecular weight of $\sim 46 \mathrm{kDa}$ (Fig. 1c; Lane 1). The specificity of the rHcADRM1 protein was determined by western blot, probing with anti-H. contortus serum or normal goat serum. A single band $\sim 46 \mathrm{kDa}$ was observed through the specific recognition of rHcADRM1 protein by anti$H$. contortus serum (Fig. 1c; Lane 2), while no band was identified via healthy goat sera (Fig. 1c; Lane 3). Meanwhile, native HcADRM1 protein derived from HcESPs was identified by rat anti-rHcADRM1 IgG as a single band of $\sim 42 \mathrm{kDa}$ (Fig. 1c; Lane 4), while no positive band was observed in the control groups (Fig. 1c; Lane 5).

\section{Differential mRNA expression in H. contortus life-cycle stages and immunolocalization}

Transcription analysis by real-time RT-PCR revealed that mRNA expression of HcADRM1 were detectable at all of the tested $H$. contortus life-cycle stages. The data demonstrated rising expression levels from the free-living stages (eggs and L3) to parasitic stages (xL3, female and male adults). Simultaneously, the highest level of HcADRM1 transcription was observed in male adults, but not in female adults (Fig. 1d). Given that the highest mRNA expression was detected in adult worms, we next investigated the localization of native HcADRM1 proteins within $H$. contortus by checking the cryosections of the adult worms. Specific red fluorescence resulting from tagging HcADRM1 proteins by rat anti-rHcADRM1 IgG was ubiquitously observed from the intracellular and cytoplasmic localization of somatic cells, particularly in the intestinal regions and the internal membrane of cuticle for both male and female adults (Fig. 1e). However, no Cy3-fluorescence was observed in the sections treated with normal rat IgG (Fig. 1e).

\section{Binding of rHcADRM1 protein to goat T cells}

Based on our preliminary LC-MS/MS analysis, we next conducted immunocytochemistry assays to verify the in vitro interaction of HcADRM1 proteins with goat T cells. Immunocytochemistry assay showed that intense red 


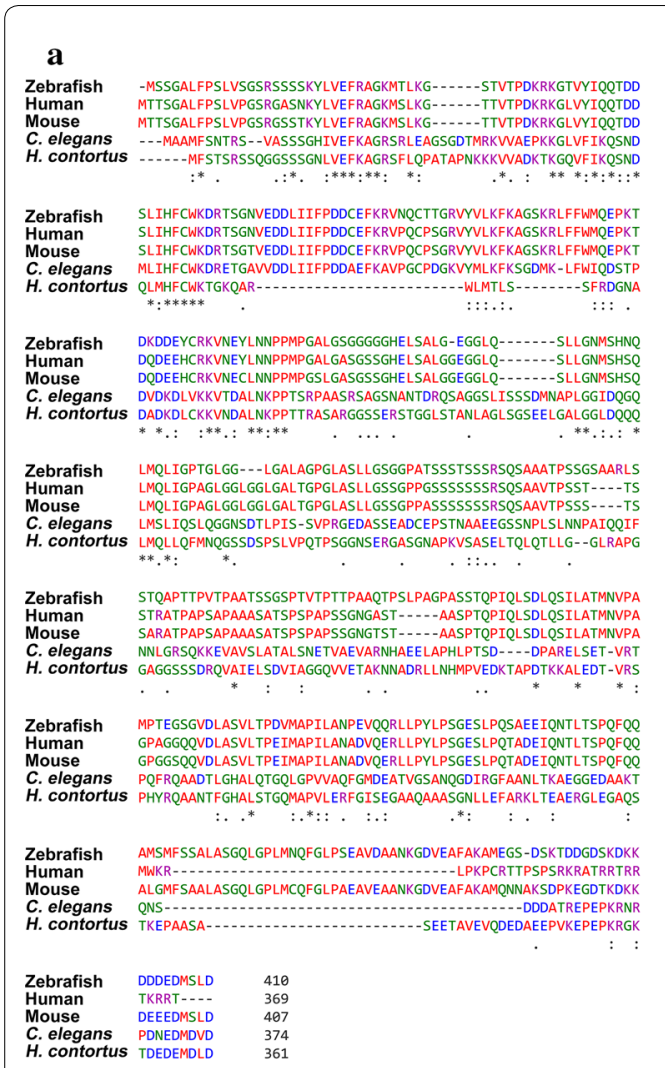

d

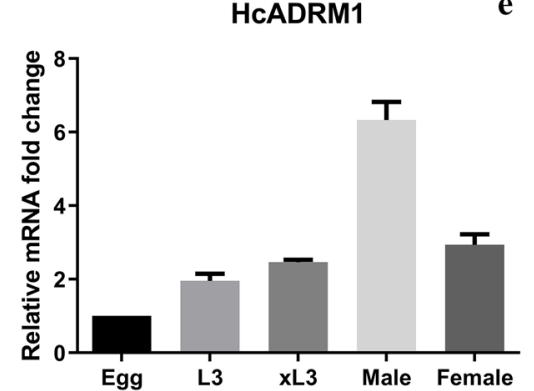

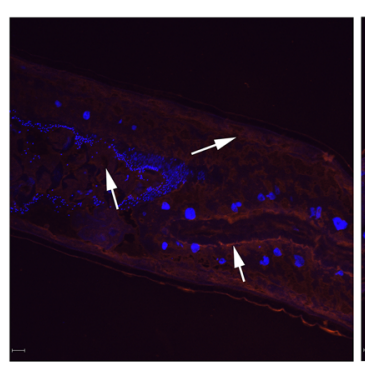

Female

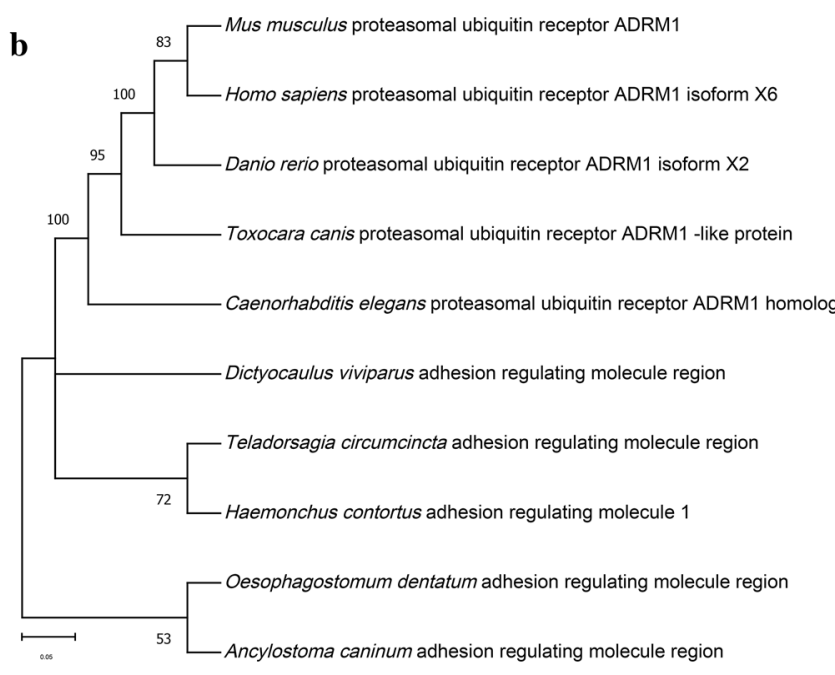

c
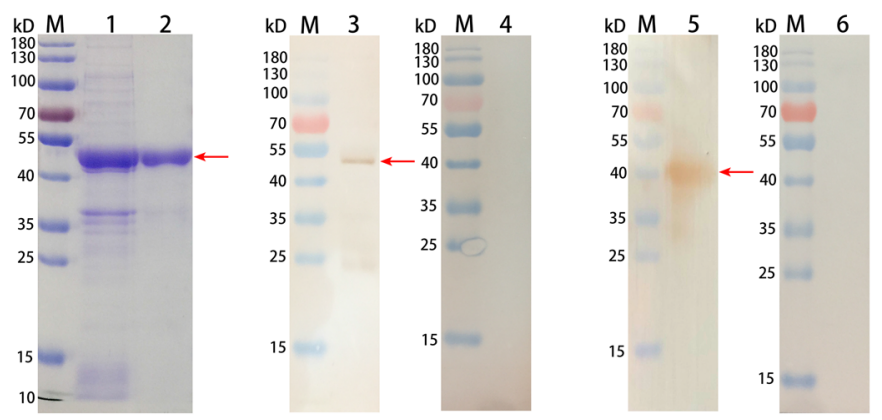

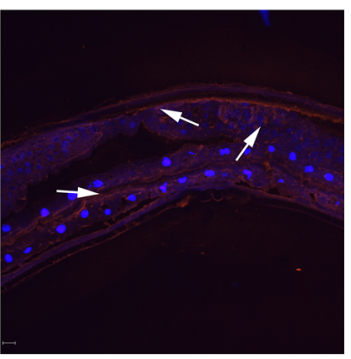

Male

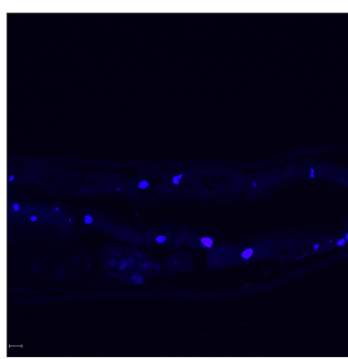

Control

Fig. 1 Molecular characterization of HCADRM1 derived from HCESPS. a Alignment of HcADRM1 amino acid sequences with other orthologues. The positions of ADRM1 family motifs are indicated above the multiple sequence alignment, containing zebrafish (XP_021325529.1), human (NP_001268366.1), mouse (NP_062796.2), C. elegans (NP_498387.2) and H. contortus (W6NB91) ADRM1 ortholog sequences retrieved from GenBank. An asterisk indicates the position with one completely conserved amino acid, while period denotes weakly conserved similarity within different groups and colon represents strongly similar conservation between groups. b Phylogenetic analysis of HCADRM1 with vertebrate and parasite orthologues. Evolutionary relationships of taxa were inferred using the Maximum Likelihood method with protein sequences including Mus musculus (NP_062796.2), Homo sapiens (XP_011526805.1), Danio rerio (XP_021325529.1), Toxocara canis (KHN87202.1), C. elegans (NP_498387.2), Dictyocaulus viviparus (KJH49054.1), T. circumcincta (PIO73930.1), H. contortus (W6NB91), Oesophagostomum dentatum (KHJ97330.1) and Ancylostoma caninum (RCN48176.1). Bootstrap support values are shown for each node. The scale-bar denotes the number of substitutions per site. c Acquisition of rHCADRM1 proteins and western blot analysis. Lanes M: protein standard ladder; Lane 1: rHCADRM1 expressed in the supernatant of cell lysates; Lane 2: SDS-PAGE analysis of purified rHCADRM1 protein; Lane 3: immunoblot analysis of rHcADRM1 using anti-H. contortus serum as primary antibody; Lane 4: immunoblot analysis of rHcADRM1 using normal goat serum (control) as primary antibody; Lane 5: immunoblot analysis of HCESPs using rat anti-rHCADRM1 IgG as primary antibody; Lane 6: immunoblot analysis of HcESPs using normal rat lgG (control) as primary antibody. $\mathbf{d}$ HCADRM1 expression in H. contortus life-cycle stages. Data are presented as the mean \pm SD. e Immunolocalization of native HcADRM1 protein in male and female adults. The immunohistochemistry assays were performed using normal rat lgG (control) or rat anti-rHcADRM1 lgG as primary antibody. Cy3-coupled fluorescence (red), along with DAPI (blue), was identified for the investigation of HcADRM1 distribution. Scale-bars: e, $200 \mu \mathrm{m}$ 
Cy3-fluorescence (resulting from tagging rHcADRM1) was observed in rHcADRM1-treated $\mathrm{T}$ cells, revealing the cytomembrane and cytoplasmic localization of rHcADRM1 (Fig. 2a, $a_{1}$ ); no red fluorescence was detected in both blank and negative control groups (Fig. $2 a, a_{2}$ and $a_{3}$ ). The results presented here further validated the positive interactions between HcADRM1 protein and host $\mathrm{T}$ cells.

\section{rHcADRM1 suppressed cell viability and induced cell apoptosis}

Given the modulatory potential of ADRM proteins on cellular development and survival, we next investigated the impact of rHcADRM1 proteins on $\mathrm{T}$ cell viability. The results of CCK- 8 determination showed that $\mathrm{T}$ cell viability was dramatically inhibited by the stimulation of $5 \mu \mathrm{g} / \mathrm{ml}$ (ANOVA: $\left.F_{(4,40)}=10.01, P=0.0045\right), 10 \mu \mathrm{g} / \mathrm{ml}$ (ANOVA: $\left.F_{(4,40)}=10.01, P=0.0004\right), 20 \mu \mathrm{g} / \mathrm{ml}$ (ANOVA: $F_{(4,40)}=10.01, P=0.0009$ ) and $40 \mu \mathrm{g} / \mathrm{ml}$ (ANOVA: $F_{(4,}$ $40)=10.01, P<0.0001)$ of rHcADRM1 proteins (Fig. 2b). Based on this finding, an Annexin V-PE/7-AAD double staining kit was employed to evaluate the pro-apoptotic potential of rHcADRM1 proteins. Flow cytometry results demonstrated that rHcADRM1 stimuli, at the tested concentrations of $10 \mu \mathrm{g} / \mathrm{ml}$ (ANOVA: $F_{(4,40)}=12.50$, $P=0.0124), \quad 20 \quad \mu \mathrm{g} / \mathrm{ml} \quad$ (ANOVA: $F_{(4,40)}=12.50$, $P<0.0001$ ) and $40 \mu \mathrm{g} / \mathrm{ml}$ (ANOVA: $F_{(4,40)}=12.50$, $P<0.0001)$ remarkably caused $\mathrm{T}$ cell apoptosis in comparison to the unstimulated group (Fig. 2c, d). Additionally, transcriptional analysis of key genes involved in apoptosis signaling pathways further validated the proapoptotic effects of rHcADRM1 proteins on host $\mathrm{T}$ cells. The treatments with 10,20 and $40 \mu \mathrm{g} / \mathrm{ml}$ of rHcADRM1 dramatically upregulated mRNA transcripts of caspase-8 (ANOVA: $F_{(4,40)}=5.557, P=0.0409, P=0.0231$ and $P=0.0002$, respectively) and caspase-3 (ANOVA: $F_{(4 \text {, }}$ ${ }_{40)}=3.296, P=0.0315, P=0.0418$ and $P=0.0054$, respectively) (Fig. 2e). Simultaneously, $20 \mu \mathrm{g} / \mathrm{ml}$ (ANOVA: $\left.F_{(4,40)}=8.265, P=0.0020\right)$ and $40 \mu \mathrm{g} / \mathrm{ml}$ (ANOVA: $F_{(4 \text {, }}$ $40)=8.265, P<0.0001)$ of rHcADRM1 proteins significantly promoted caspase- 9 transcription (Fig. 2e).

\section{rHCADRM1 protein restrained the proliferation of T cells and caused cell cycle stalling}

As apoptosis, proliferation and cell cycle were interconnected cellular movements, we next explored the modulatory potentials of rHcADRM1 stimuli on $\mathrm{T}$ cell proliferation and cell cycle. At the tested doses of 10,20 and $40 \mu \mathrm{g} / \mathrm{ml}$, flow cytometry data showed that rHcADRM1 stimuli significantly inhibited $\mathrm{T}$ cell proliferation in vitro (Fig. 3a), as indicated by the decreasing proportion of EdU ${ }^{+}$cells compared with control cells (ANOVA: $F_{(4,40)}=5.150, P=0.0482, P=0.0104$ and
$P=0.0006$, respectively) (Fig. $3 \mathrm{~b}$ ). Given that the treatments with $20 \mu \mathrm{g} / \mathrm{ml} \mathrm{rHcADRM1}$ had significant biological effects on cell viability, apoptosis and proliferation, as well as the transcription of certain key genes, we next treated T cells with $20 \mu \mathrm{g} / \mathrm{ml}$ of rHcADRM1 for cell cycle determination. Here, flow cytometry analysis with PI staining demonstrated that rHcADRM1 stimuli induced cell cycle arrest in a time-dependent manner (Fig. 3c), as indicated by the increased proportion of T cells in G1 phase at $12 \mathrm{~h}$ (ANOVA: $\left.F_{(8,96)}=17.49, P=0.0293\right), 18 \mathrm{~h}$ (ANOVA: $\left.F_{(8,96)}=17.49, P=0.0165\right)$ and $24 \mathrm{~h}$ (ANOVA: $\left.F_{(8,96)}=17.49, P=0.0028\right)$, as well as the decreased proportion of $\mathrm{T}$ cells in $\mathrm{S}$ phase at $12 \mathrm{~h}$ (ANOVA: $F_{8}$, $\left.\left.{ }_{96}\right)=17.49, P=0.0303\right), 18 \mathrm{~h}$ (ANOVA: $F_{(8,96)}=17.49$, $P=0.0480)$ and 24 h (ANOVA: $\left.F_{(8,96)}=17.49, P=0.0118\right)$ (Fig. 3d). Consistent with these findings, transcriptional analysis of key genes in G1/S checkpoints showed that mRNA transcripts of CCNE1 (t-test: $t_{(16)}=3.030$, $P=0.0080)$ and CDK2 (t-test: $t_{(16)}=2.180, P=0.0445$ ) were significantly downregulated by rHcADRM1 stimuli, while no significant transcriptional changes of CCND1 (t-test: $\left.t_{(16)}=1.748, P=0.0997\right)$, CDK4 (t-test: $\left.t_{(16)}=0.3238, P=0.7503\right)$ and CDK6 (t-test: $t_{(16)}=0.1030$, $P=0.9192$ ) were observed (Fig. 3e). Given the inhibitory effects of p21 and p27 on CDKs in Ub-mediated cell cycle progression, the transcription analysis of p21 and p27 was performed in this study. In addition, the mRNA transcripts of $I \kappa B \alpha$ as the physiological substrate of ADRM1 and its downstream inhibitor NF- $\mathrm{kB}$ were determined. Importantly, transcription of p21 (t-test: $t_{(16)}=3.665$, $P=0.0021)$, p27 (t-test: $\left.t_{(16)}=2.131, P=0.0490\right)$ and $\mathrm{I \kappa B \alpha}$ (t-test: $\left.t_{(16)}=3.154, P=0.0061\right)$ was notably enhanced by rHcADRM1 stimuli (Fig. 3e), whereas the mRNA transcript of NF-kB (t-test: $\left.t_{(16)}=2.623, P=0.0185\right)$ was significantly suppressed (Fig. 3e).

\section{Determination of cytokine secretions}

To investigate the modulatory effects of rHcADRM1 on $\mathrm{T}$ cell cytokine productions, IL-2, IL-4, IL-10, IL-17A, IFN- $\gamma$ and TGF- $\beta 1$ secretions in the cell culture supernatant were determined via ELISA assays. The data showed that the exposure of goat T cells to rHcADRM1 proteins led to the alteration of their cytokine production profiles. Intriguingly, at the tested doses of 10,20 and $40 \mu \mathrm{g} / \mathrm{ml}$, rHcADRM1 stimuli predominantly inhibited secretions of IL-4 (ANOVA: $F_{(4,40)}=3.097, P=0.0388, P=0.0279$ and $P=0.0151$, respectively), IL-10 (ANOVA: $F_{(4,}$ ${ }_{40)}=4.129, P=0.0261, P=0.0493$ and $P=0.0064$, respectively) and IFN- $\gamma$ (ANOVA: $F_{(4,40)}=4.236, P=0.0497$, $P=0.0035$ and $P=0.0117$, respectively) (Fig. $4 \mathrm{~b}, \mathrm{c}, \mathrm{e}$ ). However, all the tested doses of rHcADRM1 had no notable effects on secretions of IL-2 (ANOVA: $F_{(4,40)}=0.6883$, $P=0.4558, \quad P=0.5343, \quad P=0.8819$ and $P=0.4854$, 


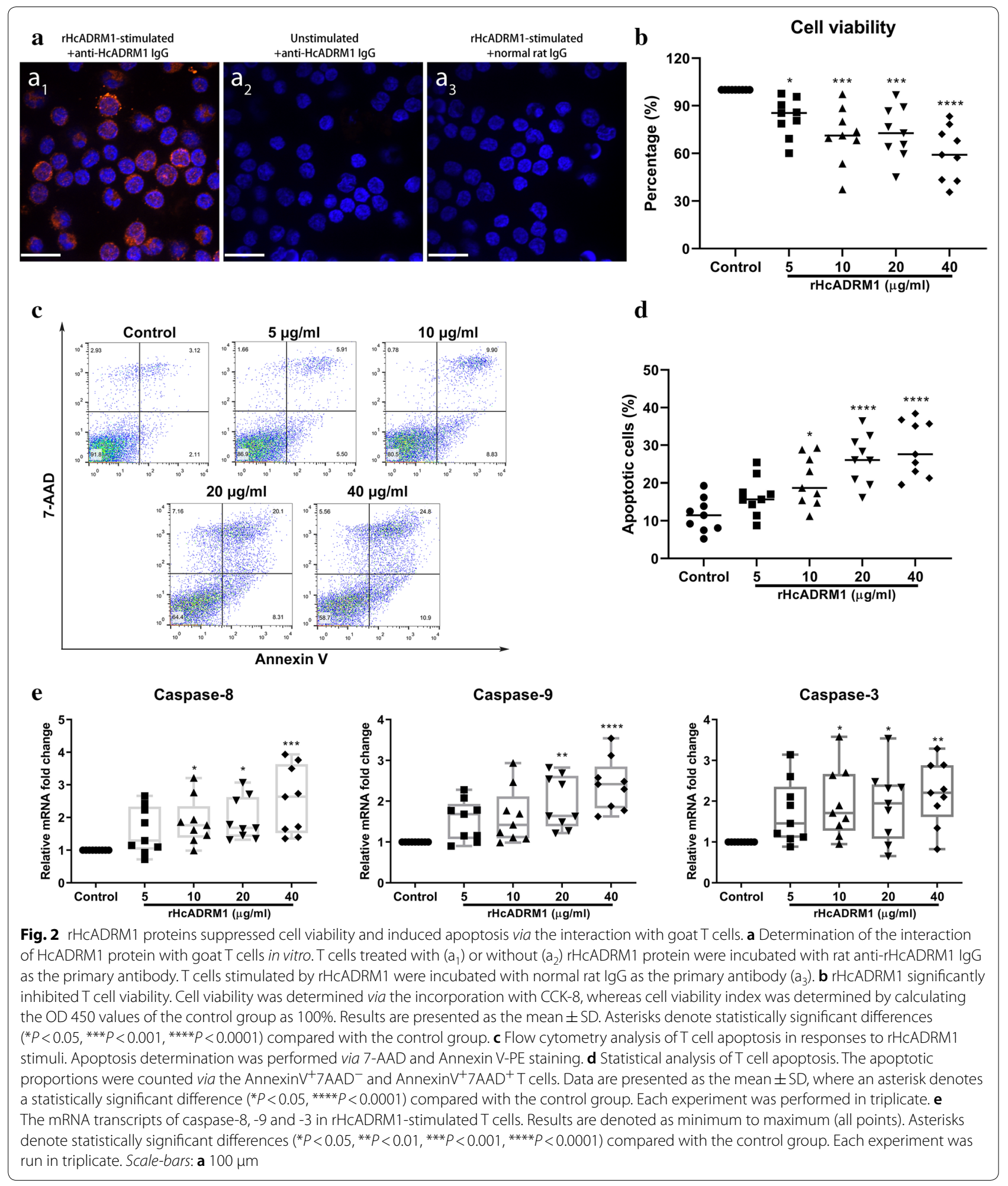

respectively), IL-17A (ANOVA: $F_{(4,40)}=0.8377$, $P=0.9966, P=0.4641, P=0.9876$ and $P=0.9967$, respectively) and TGF- $\beta 1$ (ANOVA: $F_{(4,40)}=1.480, P=0.9869$,
$P=0.9952, \quad P=0.9779$ and $P=0.2874$, respectively) production in comparison to the unstimulated group (Fig. 4a, d, f). Collectively, rHcADRM1 stimuli exerted 


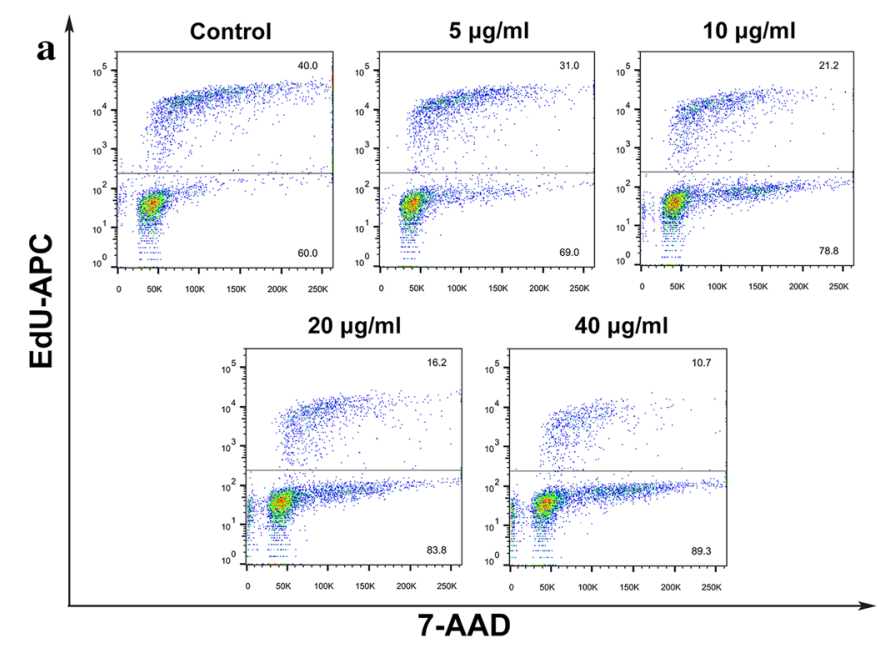

b

$\mathrm{EdU}^{+}$cells
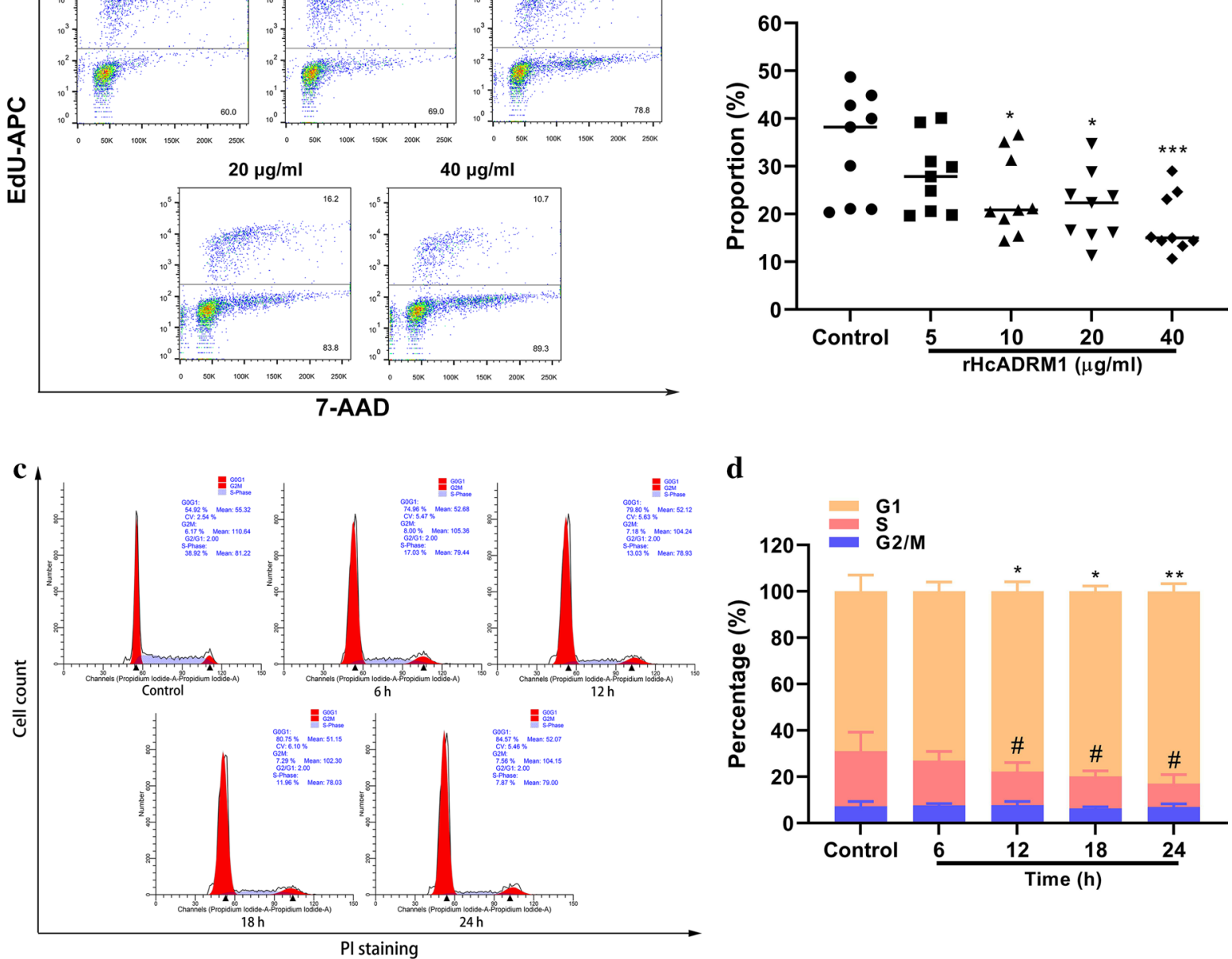

d

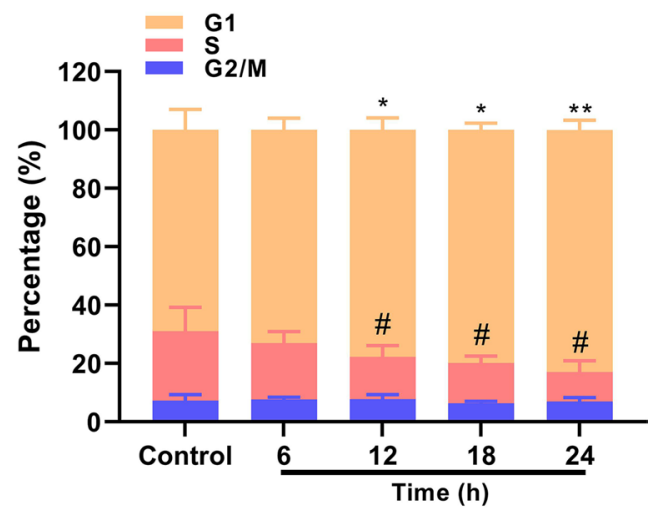

e
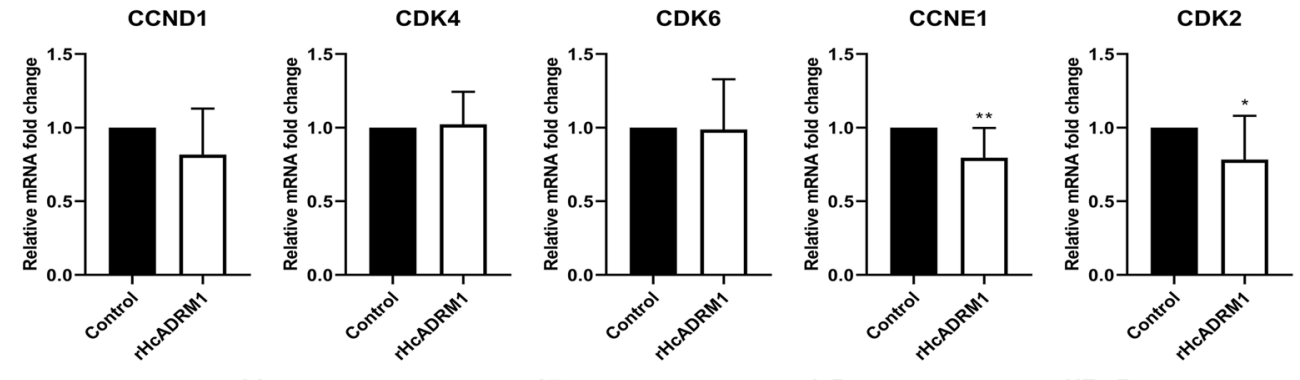

p21
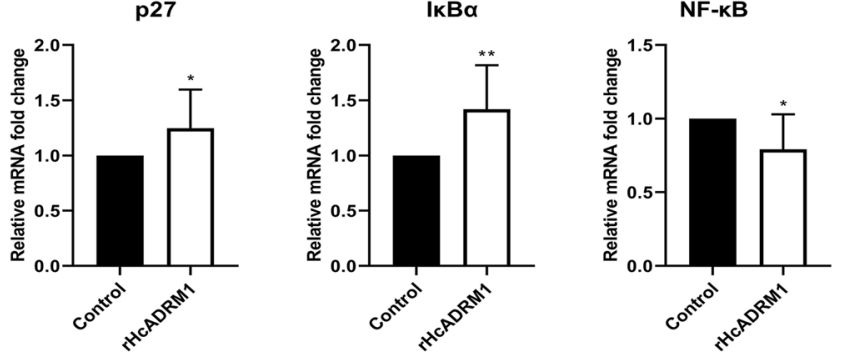
(See figure on previous page.)

Fig. 3 rHCADRM1 protein suppressed T cell proliferation and induced T cell cycle stalling at the G1 phase. a Determination of T cell proliferation in responses to rHCADRM1 stimuli. Flow cytometry analysis was performed using 7-AAD and EdU-APC double staining. b Statistical analysis of the T cell proliferation rates expressed as the proportions of EdU ${ }^{+} T$ cells. Data are presented as the mean $\pm S D$. Asterisks denote statistically significant differences ( $\left.{ }^{*} P<0.05,{ }^{* *} P<0.001\right)$ compared with the control group. $\mathbf{c} T$ cell cycle analysis in responses to rHcADRM1 stimuli. T cell cycle determination was performed using PI/RNase staining reagent by flow cytometry. $\mathbf{d}$ Statistical analysis of T cell cycle analysis. T cells incubated with $\mathrm{rHCADRM} 1$ proteins were collected every $6 \mathrm{~h}$ for flow cytometry analysis. The data demonstrated that rHcADRM1 stimuli caused cell cycle stalling at the G1 phase. Results are presented as the mean \pm SD. Asterisks for G1 phase determination $\left({ }^{*} P<0.05,{ }^{* *} P<0.01\right)$ and ${ }^{*}$ (hashtags) for $S$ phase determination $(P<0.05)$ denote statistically significant differences compared with the control group. Each experiment was run in triplicate. e Relative transcription levels of CCND1, CCNE1, CDK4/6, CDK2, p21, p27, IKBa and NF-KB in rHcADRM1-treated T cells. Data are presented as the mean \pm SD. Asterisks indicate statistically significant differences $\left({ }^{*} P<0.05,{ }^{* *} P<0.01\right)$ compared with the control group. Each experiment was run in triplicate

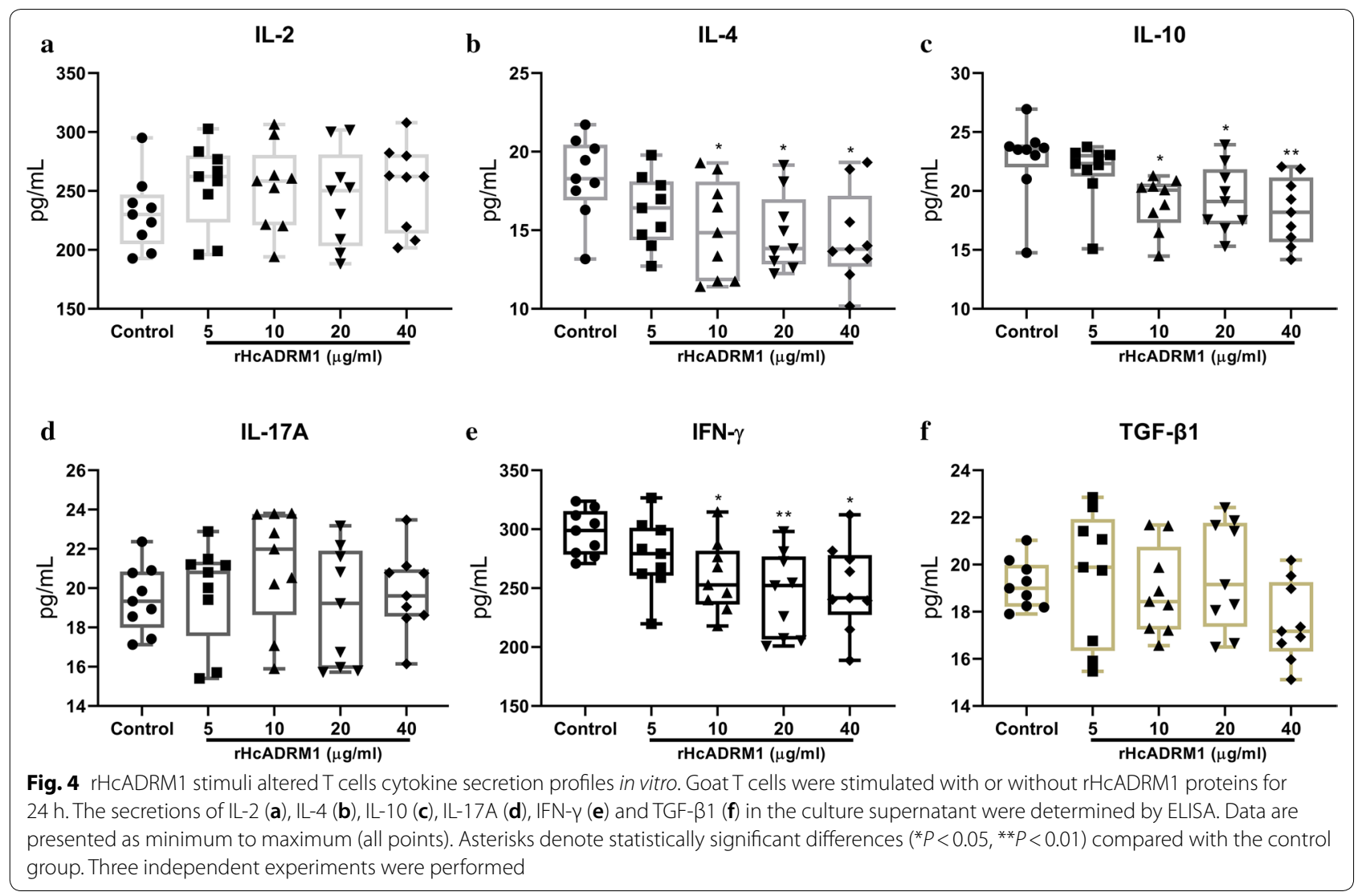

immunomodulation activities on host $\mathrm{T}$ cells via the alteration of cytokine secretion profiles.

\section{Discussion}

Much of our understanding of parasite immune evasion comes from the sophisticated and highly integrated mechanisms underlying their coexistence with hosts, e.g. parasitic helminths could release ES products or proteins into the host environment to suppress or subvert host immune responses to ensure their survival capabilities $[34,35]$. As an individual ES component derived from HcESPs that interacted with host T cells, the HcADRM1 gene was completely cloned, and the rHcADRM1 protein was successfully obtained in the present study. While ADRM1 proteins are ubiquitously distributed and highly conserved from yeasts to mammals [3, 4], HcADRM1 protein was confirmed to belong to the ADRM1 family, forming a cluster close to T. circumcincta orthologue but distant from vertebrate ADRM1 homologues based on the phylogenetic analysis. Consistent with prior identification of rHcADRM1 by sera from infected goats [16], native HcADRM1 protein from HcESPs could be appraised by specific rat anti-HcADRM1 IgG, which in turn validated our preliminary LC-MS/MS data (our 
unpublished data). Like mammalian ADRM1 as an anticancer target [13], HcADRM1 with outstanding diagnostic utility might be a favourable vaccine candidate for therapeutic prevention against $H$. contortus infection. In addition, we observed enriched cytosolic localizations of native HcADRM1 proteins within the internal cuticle and gut region of adults, probably indicating the active/passive secretions or excretions of HcADRM1 via the worm cuticle or gut [35-37]. The ADRM1 gene is ubiquitously expressed in human tissues with the highest levels in the testis [38], providing a rationale for the higher transcriptional level of the HcADRM1 gene in male adults rather than female adults of $H$. contortus. Importantly, the amounting expression of HcADRM1 during developmental life-cycle stages demonstrated the crucial role of HcADRM1 in larvae development and survival of $H$. contortus.

Although the existence of ADRM1 is required for cellular proteostasis networks involving transcriptional regulation, cell cycle, apoptosis and immune response, determining the accurate biological roles of ADRM1 individually is complicated due to its functional redundancy shared with RPN1 and RPN10 and a complex structurefunction relationship with Uch37 [39-41]. Moreover, to our knowledge, currently there are no data related to the regulation and physiological functions of parasite ADRM1 proteins. Here, we observed that exogenous rHcADRM1 protein bound positively to host $\mathrm{T}$ cells in vitro and showed cytomembrane and cytoplasmic localizations. Based on previous studies which demonstrated the functional relationships between proteostatic stress and cellular survival [42], we next validated the modulatory impacts of rHcADRM1 on cell viability and apoptosis of host effector cells as potential immunomodulator and external stimuli. Unsurprisingly, the treatments of rHcADRM1 proteins dramatically inhibited $T$ cell viability and induced cell apoptosis. As for the cell apoptosis pathway, initiator caspases like caspase- 8 and caspase- 9 triggered by apoptotic signals oligomerize and become cleaved, and lead to the activation of downstream targets such as caspase-3 [43]. Subsequently, these effector caspases in turn induce apoptosis via cleavage and modifications of target proteins [44]. The oligomerization of death receptors triggers the activation of caspase- 8 in extrinsic apoptosis pathway, whereas cytochrome $C$ released from damaged mitochondria initiates caspase- 9 activation in intrinsic apoptosis pathway [43, 45]. Intriguingly, rHcADRM1 stimuli notably advanced caspases-8, -9 and -3 transcription, indicating the potential mechanism underlying HcADRM1-induced intrinsic and extrinsic apoptosis of $\mathrm{T}$ cells.

For cell apoptosis, proliferation and cell cycle are fundamental and ultimately linked cellular events [46]; we further verified potential immunomodulatory effects of rHcADRM1 on cell proliferation and revealed that rHcADRM1 protein eminently restrained $\mathrm{T}$ cell growth in vitro. In eukaryotic cells, cell cycles are controlled by the G1/S checkpoint via CDK2-cyclin E or CDK4/6cyclin D kinase compositions, alongside the $G 2 / \mathrm{M}$ checkpoint via cyclin B-CDK1 kinase complex [47]. In most cases, the Ub proteasome system is accountable for protein homeostasis and extensively associated with the removal of sabotaged checkpoint proteins to ensure proper timing of cell cycle phase to the next [2]. In this study, our flow cytometry data suggested that rHcADRM1 caused cell cycle stalling at the G1 phase, but not the G2/M phase. Furthermore, the transition through the G1 phase into $S$ phase in T cells was prevented by rHcADRM1 via the downregulation of CCNE1 and CDK2 transcriptions and the upregulation of $\mathrm{p} 21$ and p27 transcriptions. Collectively, the Ub proteasome pathway, along with nuclear export, is vital for the timely and acute degradation/destruction of all accumulated regulatory proteins in cell division process [48]. Together with Uch37, ADRM1 protein is imperative for the precise cell cycle progress and failure of stable expression of Uch37 and ADRM1 leads to G1 arrest [49]. Therefore, the external stimuli of exogenous rHcADRM1 may hamper the ADRM1-Uch37 interaction in host T cells, disrupt the balance of cell cycle protein degradation and interfere with ADRM1 substrate IKB $\alpha$ signaling along with its downstream effectors $[49,50]$. The latter was demonstrated by the transcriptional analysis of $I \kappa B \alpha$ and NF- $k B$ in the present study. Taken together, this could be the mechanistic study of how HcADRM1 targets the regulation of host $\mathrm{T}$ cell cycle, apoptosis and proliferation.

Immunomodulation mediated via ES proteins of parasitic helminths is generally characterized with a plethora of attributes: blocking pro-inflammatory and Th1 cytokines such as IL- 2 and IFN- $\gamma$; inducing antiinflammatory cytokines including IL-10 and TGF- $\beta$; modulating Th2 responses like the secretion of IL-4; and regulating Th17 and Treg responses [35, 51]. Consistent with these findings, rHcADRM1 stimuli significantly suppressed the secretion of IL-4, but not IL-2, indicating the critical controls of HcADRM1 on Th2 responses probably via the induction of Th2 apoptosis. In addition, our data revealed that IFN- $\gamma$ and IL-10 secretion was downregulated by rHcADRM1 stimuli. I $\mathrm{B} \alpha \alpha$ is one of the physiologic substrates of ADRM1, as well as an essential binding subunit of NF- $\mathrm{KB}[50,52]$. Thus, the decreased levels of IкB $\alpha$ proteasomal degradation resulting from external rHcADRM1 stimuli may inhibit the NF- $\mathrm{kB}$ pathway connected with inflammation reactions and cell growth, thereby may in turn inhibit IFN- $\gamma$, IL-4 and IL-10 secretions [53, 54]. Taken 
together, we hereby identified HcADRM1 as an immunomodulator at the parasite-host interface via the inhibition of $\mathrm{T}$ cell survival and growth. However, due to the absence of available goat immune reagents, we only validated the transcription levels of several key molecules involving apoptosis, proliferation and cell cycle in this study. More detailed mechanistic networks at the protein level, alongside associated pathways, merit further investigation. Clearly, future exploration is indispensable to address the potential role of HcADRM1 for prophylactic therapy in anti- $H$. contortus vaccine development.

\section{Conclusions}

We identified and characterized a novel ADRM1 orthologue with immunomodulatory utility derived from HcESPs. Employing the bioinformatic approaches integrated with immunological bioassays, we demonstrated that HcADRM1 protein is expressed in all developmental stages of $H$. contortus and could interact with host key effector cells and interfere with a plethora of cellular events via repression of $\mathrm{T}$ cell viability and proliferation, facilitation of $\mathrm{T}$ cell apoptosis, stalling of dynamic $\mathrm{T}$ cell cycle, disruption of pathway signaling and alteration of cytokine production profiles. To our knowledge, this is a proteomic-guided comprehensive investigation for parasite orthologues of the ADRM1 family. The results of this study may improve our understanding of ADRM1 proteins from parasitic nematodes and continue to illustrate the diverse range of immunomodulatory activities of ES proteins.

\section{Supplementary information}

Supplementary information accompanies this paper at https://doi. org/10.1186/s13071-020-04297-7.

Additional file 1: Figure S1. Goat T cell sorting by MACS. The purity of isolated T cells was validated via flow cytometry to be above $95 \%$ as indicated before (a) and after (b) MACS sorting.

Additional file 2: Table S1. Primer sequences for HCADRM1 transcription analysis. Table S2. Primer sequences for the transcription analysis of apoptosis and cell cycle.

\footnotetext{
Abbreviations

Ub: ubiquitin; RP: regulatory particle; LC-MS/MS: liquid chromatography-tandem mass spectrometry; ADRM1: adhesion-regulating molecule 1; IL: interleukin; TGF: transforming growth factor; IFN: interferon; PBMCs: peripheral blood mononuclear cells; ES: excretory-secretory; HcESPs: Haemonchus contortus ES proteins; IkBa: NF-kB inhibitor alpha; CDK: cyclin-dependent kinase; CCK-8: cell counting kit-8; ConA: concanavalin A; 7-AAD: 7-aminoactinomycin D; EdU: 5-ethynyl-2'-deoxyuridine; NF-kB: nuclear factor kappa B; pAb: polyclonal antibody.
}

Acknowledgements

We thank Miss Ai-ling Tian for valuable and constructive suggestions.

\section{Authors' contributions}

$\mathrm{XRL}$ and $M M L$ conceptualized and designed the study. $\mathrm{XRL}$ and $\mathrm{CL}$ directed and supervised the study. MML performed data analysis and drafted the manuscript. CL edited and revised the manuscript. XWT, YZ and WJW collected blood samples and helped in flow cytometry analysis. KA and ME performed and interpreted the computational analysis. RFY, XLX and XKS provided all research resources and commented on project design. All authors read and approved the final manuscript.

\section{Funding}

This study received financial support from the National Key Research and Development Program of China (Grant No. 2017YFD0501200), the Policy Guidance Project of Jiangsu Province for International Scientific and Technological Cooperation (Grant No. BZ2019013), and the National Key Basic Research Program (973 Program) of P.R. China (Grant No. 2015CB150300).

\section{Availability of data and materials}

The datasets supporting the conclusions of this article are included within the article and its additional files.

\section{Ethics approval and consent to participate}

All protocols had been reviewed prior to experiments with provincial approval (SYXK (SU) 2010-0005). All animal studies were carried out to comply with the Guidelines of the Chinese Animal Welfare Council. Daily health conditions were monitored throughout the experiments.

\section{Consent for publication}

Not applicable.

\section{Competing interests}

The authors declare that they have no competing interests.

\section{Author details}

${ }^{1}$ MOE Joint International Research Laboratory of Animal Health and Food Safety, College of Veterinary Medicine, Nanjing Agricultural University, Nanjing 210095, Jiangsu, People's Republic of China. ${ }^{2}$ Animal Biosciences and Biotechnology Laboratory, Beltsville Agricultural Research Center, Agricultural Research Service, USA Department of Agriculture, Beltsville, MD 20705, USA.

Received: 16 March 2020 Accepted: 21 May 2020

Published online: 18 August 2020

\section{Reference}

1. Murata S, Yashiroda H, Tanaka K. Molecular mechanisms of proteasome assembly. Nat Rev Mol Cell Biol. 2009;10:104-15.

2. Amm I, Sommer T, Wolf DH. Protein quality control and elimination of protein waste: the role of the ubiquitin-proteasome system. Biochim Biophys Acta. 2014;1843:182-96.

3. Schreiner P, Chen X, Husnjak K, Randles L, Zhang N, Elsasser S, et al. Ubiquitin docking at the proteasome through a novel pleckstrin-homology domain interaction. Nature. 2008;453:548-52.

4. Husnjak K, Elsasser S, Zhang N, Chen X, Randles L, Shi Y, et al. Proteasome subunit Rpn13 is a novel ubiquitin receptor. Nature. 2008;453:481-8.

5. Hamazaki J, lemura S, Natsume T, Yashiroda H, Tanaka K, Murata S. A novel proteasome interacting protein recruits the deubiquitinating enzyme UCH37 to $26 S$ proteasomes. EMBO J. 2006;25:4524-36.

6. Qiu XB, Ouyang SY, Li CJ, Miao S, Wang L, Goldberg AL. hRpn13/ADRM1/ GP110 is a novel proteasome subunit that binds the deubiquitinating enzyme, UCH37. EMBO J. 2006;25:5742-53.

7. Yao T, Song L, Xu W, DeMartino GN, Florens L, Swanson SK, et al. Proteasome recruitment and activation of the Uch37 deubiquitinating enzyme by Adrm1. Nat Cell Biol. 2006;8:994-1002.

8. Lu X, Nowicka U, Sridharan V, Liu F, Randles L, Hymel D, et al. Structure of the Rpn13-Rpn2 complex provides insights for Rpn13 and Uch37 as anticancer targets. Nat Commun. 2017;8:15540.

9. Fejzo MS, Dering J, Ginther C, Anderson L, Ramos L, Walsh C, et al. Comprehensive analysis of 20q13 genes in ovarian cancer identifies ADRM1 as amplification target. Gene Chromosome Cancer. 2008;47:873-83. 
10. Chen W, Hu X-T, Shi Q-L, Zhang F-B, He C. Knockdown of the novel proteasome subunit Adrm1 located on the 20q13 amplicon inhibits colorectal cancer cell migration, survival and tumorigenicity. Oncol Rep. 2009:21:531-7.

11. Jang SH, Park JW, Kim HR, Seong JK, Kim HK. ADRM1 gene amplification is a candidate driver for metastatic gastric cancers. Clin Exp Metast. 2014;31:727-33.

12. Fejzo MS, Anderson L, Von Euw EM, Kalous O, Avliyakulov NK, Haykinson MJ, et al. Amplification target ADRM1: role as an oncogene and therapeutic target for ovarian cancer. Int J Mol Sci. 2013;14:3094-109.

13. Song Y, Ray A, Li S, Das D, Tai Y, Carrasco R, et al. Targeting proteasome ubiquitin receptor Rpn13 in multiple myeloma. Leukemia. 2016;30:1877-86.

14. Lamerant N, Kieda C. Adhesion properties of adhesion-regulating molecule 1 protein on endothelial cells. FEBS J. 2005;272:1833-44.

15. Lu M, Tian X, Yang Z, Wang W, Tian AL, Li C, et al. Proteomic analysis revealed $T$ cell hyporesponsiveness induced by Haemonchus contortus excretory and secretory proteins. Vet Res. 2020;51:1-14.

16. Aimulajiang K, Naqvi MA, Chu W, Lu M, Tian X, Bu Y, et al. Adhesionregulating molecule from Haemonchus contortus: potential antigen for diagnosis of early infection in goats. Pathogens. 2020;9:34.

17. Wang W, Wang S, Zhang H, Yuan C, Yan R, Song X, et al. Galectin Hco-gal$\mathrm{m}$ from Haemonchus contortus modulates goat monocytes and T cell function in different patterns. Parasites Vectors. 2014;7:342.

18. Wang F, Xu L, Song X, Li X, Yan R. Identification of differentially expressed proteins between free-living and activated third-stage larvae of Haemonchus contortus. Vet Parasitol. 2016;215:72-7.

19. Li Y, Yuan C, Wang L, Lu M, Wang Y, Wen Y, et al. Transmembrane protein 147 (TMEM147): another partner protein of Haemonchus contortus galectin on the goat peripheral blood mononuclear cells (PBMC). Parasites Vectors. 2016;9:355

20. Dassanayake RP, Madsen-Bouterse SA, Truscott TC, Zhuang D, Mousel MR, Davis WC, et al. Classical scrapie prions are associated with peripheral blood monocytes and T-lymphocytes from naturally infected sheep. BMC Vet Res. 2016;12:27.

21. Madeira F, Park YM, Lee J, Buso N, GurT, Madhusoodanan N, et al. The EMBL-EBI search and sequence analysis tools APIs in 2019. Nucleic Acids Res. 2019:47:W636-41.

22. Kumar S, Stecher G, Li M, Knyaz C, Tamura K. MEGA X: Molecular Evolutionary Genetics Analysis across computing platforms. Mol Biol Evol. 2018;35:1547-9.

23. Lu M, Tian X, Yang X, Yuan C, Ehsan M, Liu X, et al. The N-and C-terminal carbohydrate recognition domains of Haemonchus contortus galectin bind to distinct receptors of goat PBMC and contribute differently to its immunomodulatory functions in host-parasite interactions. Parasites Vectors. 2017:10:409.

24. Li B, Gadahi JA, Gao W, Zhang Z, Ehsan M, Xu L, et al. Characterization of a novel aspartyl protease inhibitor from Haemonchus contortus. Parasites Vectors. 2017;10:191.

25. Ehsan M, Wang W, Gadahi JA, Hasan MW, Lu M, Wang Y, et al. The serine/ threonine-protein phosphatase 1 from Haemonchus contortus is actively involved in suppressive regulatory roles on immune functions of goat peripheral blood mononuclear cells. Front Immunol. 2018;9:1627.

26. Wang W, Wang Y, Tian X, Lu M, Ehsan M, Yan R, et al. Y75B8A. 8 (HC8) protein of Haemonchus contortus: a functional inhibitor of host IL-2. Parasites Immunol. 2019;41:e12625.

27. Tian X, Lu M, Wang W, Jia C, Muhammad E, Yan R, et al. Hc TTR: a novel antagonist against goat interleukin 4 derived from the excretory and secretory products of Haemonchus contortus. Vet Res. 2019;50:42.

28. Ehsan M, Gao W, Gadahi JA, Lu M, Liu X, Wang Y, et al. Arginine kinase from Haemonchus contortus decreased the proliferation and increased the apoptosis of goat PBMCs in vitro. Parasites Vectors. 2017;10:311.

29. Yuan C, Zhang H, Wang W, Li Y, Yan R, Xu L, et al. Transmembrane protein $63 \mathrm{~A}$ is a partner protein of Haemonchus contortus galectin in the regulation of goat peripheral blood mononuclear cells. Parasites Vectors. 2015;8:211.

30. Chang G, Liu X, Ma N, Yan J, Dai H, Roy AC, et al. Dietary addition of sodium butyrate contributes to attenuated feeding-induced hepatocyte apoptosis in dairy goats. J Agric Food Chem. 2018;66:9995-10002.

31. Gui H, Shen Z. Concentrate diet modulation of ruminal genes involved in cell proliferation and apoptosis is related to combined effects of short-chain fatty acid and $\mathrm{pH}$ in rumen of goats. J Dairy Sci. 2016;99:6627-38

32. Yao X, Ei-Samahy M, Fan $L$, Zheng $L$, Jin $Y$, Zhang G, et al. In vitro influence of selenium on the proliferation of and steroidogenesis in goat luteinized granulosa cells. Theriogenology. 2018;114:70-80.

33. Chandra Roy A, Wang Y, Zhang H, Roy S, Dai H, Chang G, et al. Sodium butyrate mitigates iE-DAP induced inflammation caused by high-concentrate feeding in liver of dairy goats. J Agric Food Chem. 2018;66:8999-9009.

34. Harris NL. Recent advances in type-2-cell-mediated immunity: insights from helminth infection. Immunity. 2017;47:1024-36.

35. Harnett W. Secretory products of helminth parasites as immunomodulators. Mol Biol Parasitol. 2014;195:130-6.

36. McNeilly TN, Nisbet AJ. Immune modulation by helminth parasites of ruminants: implications for vaccine development and host immune competence. Parasite. 2014;21:51.

37. Tundup S, Srivastava L, Harn J, Donald A. Polarization of host immune responses by helminth-expressed glycans. Ann NY Acad Sci. 2012;1253:E1-13.

38. Fagerberg L, Hallström BM, Oksvold P, Kampf C, Djureinovic D, Odeberg J, et al. Analysis of the human tissue-specific expression by genome-wide integration of transcriptomics and antibody-based proteomics. Mol Cell Proteom. 2014;13:397-406.

39. Hamazaki J, Hirayama S, Murata S. Redundant roles of Rpn10 and Rpn13 in recognition of ubiquitinated proteins and cellular homeostasis. PLoS Genet. 2015;11:e1005401.

40. Al-Shami A, Jhaver KG, Vogel P, Wilkins C, Humphries J, Davis JJ, et al. Regulators of the proteasome pathway, Uch37 and Rpn13, play distinct roles in mouse development. PLOS ONE. 2010;5:e13654.

41. Hamazaki J, Sasaki K, Kawahara H, Hisanaga S, Tanaka K, Murata S. Rpn 10mediated degradation of ubiquitinated proteins is essential for mouse development. Mol Cell Biol. 2007;27:6629-38.

42. Demishtein A, Fraiberg M, Berko D, Tirosh B, Elazar Z, Navon A. SQSTM1/ p62-mediated autophagy compensates for loss of proteasome polyubiquitin recruiting capacity. Autophagy. 2017;13:1697-708.

43. Julien O, Wells JA. Caspases and their substrates. Cell Death Differ. 2017;24:1380-9.

44. Hassan M, Watari H, AbuAlmaaty A, Ohba Y, Sakuragi N. Apoptosis and molecular targeting therapy in cancer. BioMed Res Int. 2014;2014:150845.

45. Fuchs $\mathrm{Y}$, Steller H. Programmed cell death in animal development and disease. Cell. 2011;147:742-58.

46. Evan GI, Vousden KH. Proliferation, cell cycle and apoptosis in cancer. Nature. 2001;411:342.

47. Malumbres M, Barbacid M. Cell cycle, CDKs and cancer: a changing paradigm. Nat Rev Cancer. 2009;9:153-66.

48. Skaar JR, Pagano M. Control of cell growth by the SCF and APC/C ubiquitin ligases. Curr Opin Cell Biol. 2009;21:816-24.

49. Randles L, Anchoori RK, Roden RB, Walters KJ. The proteasome ubiquitin receptor hRpn13 and its interacting deubiquitinating enzyme Uch37 are required for proper cell cycle progression. J Biol Chem. 2016;291:8773-83.

50. Mazumdar T, Gorgun FM, Sha Y, Tyryshkin A, Zeng S, Hartmann-Petersen $\mathrm{R}$, et al. Regulation of NF-KB activity and inducible nitric oxide synthase by regulatory particle non-ATPase subunit 13 (Rpn13). Proc Natl Acad Sci USA. 2010;107:13854-9.

51. Cortés A, Muñoz-Antoli C, Esteban JG, Toledo R. Th2 and Th1 responses: clear and hidden sides of immunity against intestinal helminths. Trends Parasitol. 2017;33:678-93.

52. Wu Y, Deng J, Rychahou PG, Qiu S, Evers BM, Zhou BP. Stabilization of snail by NF-KB is required for inflammation-induced cell migration and invasion. Cancer Cell. 2009;15:416-28.

53. Hanahan D, Weinberg RA. Hallmarks of cancer: the next generation. Cell. 2011;144(5):646-74.

54. Grivennikov SI, Greten FR, Karin M. Immunity, inflammation, and cancer. Cell. 2010;140:883-99.

\section{Publisher's Note}

Springer Nature remains neutral with regard to jurisdictional claims in published maps and institutional affiliations. 